\title{
Behavior of the Phase Diagrams of Potts Model on Cayley Tree for Order Three
}

\author{
S. Temir ${ }^{a}$, N. GANikHodjaev ${ }^{b}, \mathrm{~S} . \mathrm{UgUZ}^{a}, \mathrm{H} . \mathrm{AkIN}^{c}$ \\ ${ }^{a}$ Harran University, Arts and Science Faculty, Department of Mathematics, Sanliurfa, 63120, Turkey \\ ${ }^{b}$ Department of Computational and Theoretical Sciences, Faculty of Science, IIUM, 25200 Kuantan, Malaysia \\ ${ }^{c}$ Zirve University, Faculty of Education, Department of Mathematics, Gaziantep, 27260, Turkey
}

\begin{abstract}
The aim of this paper is to extend the results of Ganikhodjaev et al. to the three state Potts model with competing nearest-neighbor, prolonged next-nearest-neighbor and two-level triple neighbor interactions on a Cayley tree for order 3 and compare with the phase diagrams obtained in Temir et al. and to study modulated phases arising from the frustration effects introduced by nearest-neighbor, prolonged next-nearest-neighbor and two-level triple neighbor interactions.
\end{abstract}

DOI: 10.12693/APhysPolA.123.476

PACS:

\section{Introduction}

It is known that the Potts model has many applications due to appearance of nontrivial magnetic orderings. Consideration of the Potts model with competing interactions on Cayley tree (the Bethe lattice) is an interesting topics of statistical mechanics. A phase diagram of a model describes a morphology of phases, stability of phases, transitions from one phase to another and corresponding transitions lines. Systems exhibiting spatially modulated structures, commensurate or incommensurate with the underlying lattice, are of current interest in condensed matter physics [1]. The Ising (ANNNI) model, originally introduced by Elliot [2] to describe the sinusoidal magnetic structure of erbium, and the chiral Potts model, introduced by Ostlund [3] and Huse [4] in connection with monolayers adsorbed on rectangular substrates, have been studied extensively by a variety of techniques.

In the case of the Ising model with competing nearest-neighbor interactions and prolonged nextnearest-neighbor interactions Vannimenus [5] was able to find new modulated phases, in addition to the expected paramagnetic and ferromagnetic ones. Moreover a detailed study of its properties was carried out with essentially exact results, using rather simple numerical methods.

A Potts model as a generalization of the Ising model on the Cayley tree with competing interactions appeared in a pioneering work of Vannimenus [5] has recently been studied extensively (see [6-9]). On the Cayley tree one can consider two types of next-nearest-neighbors (triple neighbors): prolonged and one-level next-nearestneighbors (respectively two-level triple neighbors).

In this paper, we are going to study the phase diagram for the Potts model on a Cayley tree for order 3 with competing nearest-neighbor interactions $J_{n n}$, prolonged next-nearest neighbor interactions $J_{p n}$ and two level triple neighbor interactions $J_{t t}$. At vanishing temperature, the phase diagram is fully determined for all values and signs of $J_{n n}, J_{p n}$ and $J_{t t}$. The aim of this paper is to extend the results of [6], [7] and [8] to the Potts model with competing nearest- neighbor, prolonged next-nearest-neighbor and two-level triple neighbor interactions.

\section{The model and basic equations}

Now, we give some basic definitions of the Cayley tree and our using model as follows. A Cayley tree $\Gamma^{k}$ of order $k \geq 1$ is an infinite tree, i.e., a graph without cycles with exactly $k+1$ edges issuing from each vertex. Let denote the Cayley tree as $\Gamma^{k}=(V, \Lambda)$, where $V$ is the set of vertices of $\Gamma^{k}, \Lambda$ is the set of edges of $\Gamma^{k}$. For a fixed $x_{0} \in \mathrm{V}$ we set $W_{n}=\left\{x \in V \mid d\left(x, x_{0}\right)=n\right\}$,

$$
V_{n}=\left\{x \in V \mid d\left(x, x_{0}\right) \leq n\right\}=\bigcup_{i=0}^{n} W_{i}
$$

and $L_{n}$ denotes the set of edges in $V_{n}$. The fixed vertex $x_{0}$ is called the 0 -th level and the vertices in $W_{n}$ are called the $n$-th level.

For the sake of simplicity we put $|x|=d\left(x, x_{0}\right), x \in \mathrm{V}$. Two vertices $x$ and $y, x, y \in \mathrm{V}$ are called nearest-neighbors if there exists an edge $l \in \Lambda$ connecting them, which is denoted by $l=\langle x, y\rangle$. The distance $d(x, y), x, y \in \mathrm{V}$, on the Cayley tree $\Gamma^{k}$, is the number of edges in the shortest path from $x$ to $y$. Two vertices $x, y \in \mathrm{V}$ are called the next-nearest-neighbors if $d(x, y)=2$. The next-nearest-neighbor vertices $x$ and $y$ are called prolonged next-nearest-neighbors if $|x| \neq|y|$ and is denoted by $\rangle \widetilde{x, y}\langle$. The next-nearest-neighbor vertices $\mathrm{x}$ and $\mathrm{y}$ are called prolonged next-nearest-neighbors if $|x|=|y|$ and is denoted by $\rangle x, y\langle$. The next-nearest-neighbor vertices $x, y \in \mathrm{V}$ that are not prolonged are called one-level next-nearest-neighbors since $|x|=|y|$ and are denoted by $\rangle x, y\langle$. Three vertices $x, y$ and $z$ are called a triple of neighbors and they are denoted by $\langle x, y, z\rangle$, if $\langle x, y\rangle$, $\langle y, z\rangle$ are nearest neighbors. The triple of vertices $x, y, z$ is called prolonged if $x \in W_{n}, y \in W_{n+1}$ and $z \in W_{n+2}$ for some nonnegative integer $n$ and is denoted by $\langle x, z\rangle$. 
In this paper, we consider a semi-infinite Bethe lattice $\Gamma_{+}^{3}$ of order 3, i.e. an infinite graph without cycles with 3 edges issuing from each vertex except for $x^{0}$ which has only 3 edges. We plot the phase diagrams corresponding to some given parameters of model. We will compare our models with previous phase regions obtained in [6-9].

\subsection{The Model}

For Potts model with spin values in $\Phi=\langle 1,2,3\rangle$ the relevant Hamiltonian with competing nearest-neighbor, prolonged next-nearest-neighbor and two-level triple interactions has the form

$$
\begin{aligned}
& H(\sigma)=-J_{t t} \sum_{\langle\overline{x, y, z}\rangle} \delta_{\sigma(x) \sigma(y) \sigma(z)} \\
& -J_{p n} \sum_{\langle\widetilde{x, y}\langle} \delta_{\sigma(x) \sigma(y)} J_{n n} \sum_{\langle x, y\rangle} \delta_{\sigma(x) \sigma(y)},
\end{aligned}
$$

where $J_{t t}, J_{p n}, J_{n n} \in \mathrm{IR}$ are coupling constants and $\delta$ is the Kronecker symbol. Here, the generalized Kronecker's symbol $\delta_{\sigma(x) \sigma(y) \sigma(z)}$ is

$$
\begin{gathered}
\delta_{\sigma(x) \sigma(y) \sigma(z)}= \begin{cases}1, & \sigma(x)=\sigma(y)=\sigma(z), \\
0, & \text { otherwise. }\end{cases} \\
\text { 2.2 Recurrence equations }
\end{gathered}
$$

In order to produce the recurrent equations, we consider the relation of the partition function on $V_{n}$ to the partition function on subsets of $V_{n-1}$. Given the initial conditions on $V_{1}$, the recurrence equations indicate how their infiuence propagates down the tree. In order to produce the recurrent equations, we consider the relation of the partition function on $V_{n}$ to the partition function on subsets of $V_{n-1}$. Given the initial conditions on $V_{1}$, the recurrence equations indicate how their influence propagates down the tree. Let

$$
Z^{(n)}\left(\begin{array}{ccc}
i_{1} & i_{2} & i_{3} \\
i_{0}
\end{array}\right)
$$

be a partition function on $V_{n}$ where the spin in the root $x^{0}$ is where the spin in the root $x^{0}$ is $i_{0}$ and the three spins in the proceeding ones are $i_{1}, i_{2}$, and $i_{3}$, respectively.

There are $3^{4}=81$ different partition functions

$$
Z^{(n)}\left(\begin{array}{ccc}
i_{1} & i_{2} & i_{3} \\
i_{0}
\end{array}\right)
$$

and the partition function $Z(n)$ in volume $V n$ can be written as follows:

$$
Z^{(n)}=\sum_{i_{0}, i_{1}, i_{2}, i_{3}=1}^{3} Z^{(n)}\left(\begin{array}{cc}
i_{1} & i_{2} \\
i_{3} \\
i_{0}
\end{array}\right),
$$

where $i_{1}, i_{0}, i_{2}, i_{3} \in\langle 1,2,3\rangle$. Next, if it is calculated then we have the following equations

$$
\begin{aligned}
& Z^{(n)}\left(\begin{array}{c}
111 \\
1
\end{array}\right)=a^{9} c^{3} Z^{(n)}(1,1) Z^{(n)}(1,1) Z^{(n)}(1,1), \\
& Z^{(n)}\left(\begin{array}{c}
112 \\
1
\end{array}\right)=a^{3} c^{2} Z^{(n)}(1,1) Z^{(n)}(1,1) Z^{(n)}(1,2),
\end{aligned}
$$

$$
\begin{aligned}
& Z^{(n)}\left(\begin{array}{c}
113 \\
1
\end{array}\right)=a^{3} c^{2} Z^{(n)}(1,1) Z^{(n)}(1,1) Z^{(n)}(1,3), \\
& Z^{(n)}\left(\begin{array}{c}
121 \\
1
\end{array}\right)=a^{3} c^{2} Z^{(n)}(1,1) Z^{(n)}(1,1) Z^{(n)}(1,2), \\
& Z^{(n)}\left(\begin{array}{c}
122 \\
1
\end{array}\right)=c Z^{(n)}(1,1) Z^{(n)}(1,2) Z^{(n)}(1,2), \\
& Z^{(n)}\left(\begin{array}{c}
123 \\
1
\end{array}\right)=c Z^{(n)}(1,1) Z^{(n)}(1,2) Z^{(n)}(1,2), \\
& Z^{(n)}\left(\begin{array}{c}
131 \\
1
\end{array}\right)=a^{3} c^{2} Z^{(n)}(1,1) Z^{(n)}(1,2) Z^{(n)}(1,2), \\
& Z^{(n)}\left(\begin{array}{c}
222 \\
1
\end{array}\right)=Z^{(n)}(1,2) Z^{(n)}(1,2) Z^{(n)}(1,2) \\
& Z^{(n)}\left(\begin{array}{c}
222 \\
2
\end{array}\right)=a^{9} c^{2} Z^{(n)}(2,2) Z^{(n)}(2,2) Z^{(n)}(2,2), \\
& Z^{(n)}\left(\begin{array}{c}
333 \\
333 \\
2
\end{array}\right)=a^{9} c^{2} Z^{(n)}(3,3) Z^{(n)}(3,3) Z^{(n)}(3,3) . \\
& \left(\begin{array}{c}
111 \\
2
\end{array}\right)=Z^{(n)}(2,1) Z^{(n)}(2,1) Z^{(n)}(2,1),
\end{aligned}
$$

It is reasonable, though, to assume that the different branches are equivalent, as is usually done for models on trees. We can show that there are only five independent variables, namely $Z^{(n)}\left(\begin{array}{c}111 \\ 1\end{array}\right), Z^{(n)}\left(\begin{array}{c}222 \\ 1\end{array}\right), Z^{(n)}\left(\begin{array}{c}111 \\ 2\end{array}\right)$, $Z^{(n)}\left(\begin{array}{c}222 \\ 2\end{array}\right), Z^{(n)}\left(\begin{array}{c}333 \\ 2\end{array}\right)$, and introduce five new variables $u_{1}^{(n)}=\sqrt[3]{Z^{(n)}\left(\begin{array}{c}111 \\ 1\end{array}\right)}, u_{2}^{(n)}=\sqrt[3]{Z^{(n)}\left(\begin{array}{c}222 \\ 1\end{array}\right)}$, $u_{3}^{(n)}=\sqrt[3]{Z^{(n)}\left(\begin{array}{c}111 \\ 2\end{array}\right)}, u_{4}^{(n)}=\sqrt[3]{Z^{(n)}\left(\begin{array}{c}222 \\ 2\end{array}\right)}, u_{5}^{(n)}=$ $\sqrt[3]{Z^{(n)}\left(\begin{array}{c}333 \\ 2\end{array}\right)}$, we can show new calculations as follows 


$$
\begin{aligned}
& u_{1}^{(n+1)}=a^{3} c\left[\sum_{i=0}^{3}\left(\begin{array}{l}
3 \\
i
\end{array}\right)\left(b u_{1}^{(n)}\right)^{3-i}+\left(2 u_{2}^{(n)}\right)^{i} a^{\frac{3 i(i-3)}{2}}\right] \\
& u_{2}^{(n+1)}=\sum_{i=0}^{3}\left(\begin{array}{c}
3 \\
i
\end{array}\right)\left(u_{3}^{(n)}\right)^{i} b^{i} \\
& \times\left[\sum_{j=0}^{3-i}\left(\begin{array}{c}
3-i \\
j
\end{array}\right)\left(u_{4}^{(n)}\right)^{j}\left(u_{5}^{(n)}\right)^{3-i-j} a^{\frac{3 j(j-3)}{2}}\right] \\
& u_{3}^{(n+1)}=\sum_{i=0}^{3}\left[\left(u_{1}^{(n)}\right)^{3-i}\left(u_{2}^{(n)}\right)^{i}(b+1)^{i} a^{\frac{3 i(i-3)}{2}}\right] \\
& u_{4}^{(n+1)}=a^{3} c\left\{\begin{array}{l}
3 \\
i=0
\end{array}\left(\begin{array}{c}
3 \\
i
\end{array}\right)\left(b u_{4}^{(n)}\right)^{i} a^{\frac{3 i(i-3)}{2}}\right. \\
& \left.\times \sum_{j=0}^{3-i}\left[\left(\begin{array}{c}
3-i \\
j
\end{array}\right)\left(u_{3}^{(n)}\right)^{j}\left(u_{5}^{(n)}\right)^{3-i-j}\right]\right\} \\
& u_{5}^{(n+1)}=\sum_{i=0}^{3}\left(\begin{array}{c}
3 \\
i
\end{array}\right)\left(u_{5}^{(n)}\right)^{i} b^{i} \\
& \times\left[\sum_{j=0}^{3-i}\left(\begin{array}{c}
3-i \\
j
\end{array}\right)\left(u_{4}^{(n)}\right)^{j}\left(u_{3}^{(n)}\right)^{3-i-j} a^{\frac{3 j(j-3)}{2}}\right],
\end{aligned}
$$

where $c=\exp \left(\alpha^{-1}\right), \quad b=\exp \left(-\alpha^{-1} \beta\right)$ and $a=$ $\exp \left((3 \alpha)^{-1} \gamma\right)$.

The total partition function is given in terms of $\left(u_{i}\right)$ by

$$
\begin{aligned}
& Z^{(n)}=u_{1}^{(n)^{3}}+2\left(u_{3}^{(n)^{3}}+u_{4}^{(n)^{3}}+u_{5}^{(n)^{3}}\right)+6 a^{-3} u_{1}^{(n)} u_{2}^{(n)} \\
& +12 a^{-3} u_{1}^{(n)} u_{2}^{(n)^{2}}+8 u_{2}^{(n)^{3}}+6 a^{-3} u_{3}^{(n)^{2}} u_{4}^{(n)}+6 u_{3}^{(n)^{2}} u_{5}^{(n)} \\
& +7 a^{-3} u_{3}^{(n)} u_{4}^{(n)^{2}}+12 a^{-3} u_{3}^{(n)} u_{4}^{(n)} u_{5}^{(n)}+6 u_{3}^{(n)} u_{5}^{(n)^{2}} \\
& +5 a^{-3} u_{4}^{(n)^{2}} u_{5}^{(n)}+6 a^{-3} u_{4}^{(n)} u_{5}^{(n)^{2}}
\end{aligned}
$$

We note that, in the paramagnetic phase (high symmetry phase), $u_{1}^{(n)}=u_{4}^{(n)}, u_{2}^{(n)}=u_{3}^{(n)}=u_{5}^{(n)}$. For discussing the phase diagram, the following choice of reduced variables is convenient (see Refs. [6-9]):

$$
\begin{aligned}
& x=\frac{2 u_{2}+u_{3}+u_{5}}{u_{1}+u_{4}}, \quad y_{1}=\frac{u_{1}-u_{4}}{u_{1}+u_{4}}, \\
& y_{2}=\frac{u_{2}-u_{3}}{u_{1}+u_{4}}, \quad y_{3}=\frac{u_{2}-u_{5}}{u_{1}+u_{4}} .
\end{aligned}
$$

The variable $x$ is just a measure of the frustration of the nearest-neighbor bonds and is not an order parameter like $y_{1}, y_{2}, y_{3}$. The relations now have following form:

$$
\begin{aligned}
x^{\prime}=\frac{A_{1}\left(x, y_{1}, y_{2}, y_{3}\right)}{D\left(x, y_{1}, y_{2}, y_{3}\right)}, & y_{1}^{\prime}=\frac{A_{2}\left(x, y_{1}, y_{2}, y_{3}\right)}{D\left(x, y_{1}, y_{2}, y_{3}\right)} \\
y_{2}^{\prime}=\frac{A_{3}\left(x, y_{1}, y_{2}, y_{3}\right)}{D\left(x, y_{1}, y_{2}, y_{3}\right)}, & y_{3}^{\prime}=\frac{A_{4}\left(x, y_{1}, y_{2}, y_{3}\right)}{D\left(x, y_{1}, y_{2}, y_{3}\right)},
\end{aligned}
$$

where we obtain the functions $A_{1}, A_{2}, A_{3}, A_{4}$ and $D$ as follows: $A_{1}=2\left[b^{3}\left(x+y_{3}-3 y_{2}\right)^{3}+6 a^{-3} b^{2}\left(1-y_{1}\right)(x+\right.$ $\left.y_{3}-3 y_{2}\right)^{2}+3 b^{2}\left(x+y_{3}-3 y_{2}\right)^{2}\left(x+y_{2}-3 y_{3}\right)+8\left(1-y_{1}\right)^{3}+$ $12 b a^{-3}\left(1-y_{1}\right)\left(x+y_{3}-3 y_{2}\right)\left(x+y_{2}-3 y_{3}\right)+3 b\left(x+y_{3}-\right.$ $\left.3 y_{2}\right)\left(x+y_{2}-3 y_{3}\right)^{2}+12 b a^{-3}\left(1-y_{1}\right)^{2}\left(x+y_{3}-3 y_{2}\right)+$ $12 a^{-3}\left(1-y_{1}\right)^{2}\left(x+y_{2}-3 y_{3}\right)+6 a^{-3}\left(1-y_{1}\right)\left(x+y_{2}-\right.$ $\left.\left.3 y_{3}\right)^{2}+\left(x+y_{2}-3 y_{3}\right)^{3}\right]+\left[8\left(1+y_{1}\right)^{3}+12 a^{-3}\left(1+y_{1}\right)^{2}(b+\right.$ 1) $\left(x+y_{2}+y_{3}\right)+6 a^{-3}\left(1+y_{1}\right)(b+1)^{2}\left(x+y_{2}+y_{3}\right)^{2}+(1+$ b) $\left.{ }^{3}\left(x+y_{2}+y_{3}\right)^{3}\right]+\left[\left(x+y_{3}-3 y_{2}\right)^{3}+6 a^{-3}\left(1-y_{1}\right)(x+\right.$ $\left.y_{3}-3 y_{2}\right)^{2}+12 a^{-3}\left(1-y_{1}\right)^{2}\left(x+y_{3}-3 y_{2}\right)+3 b\left(x+y_{3}-\right.$ $\left.3 y_{2}\right)^{2}\left(x+y_{2}-3 y_{3}\right)+12 b a^{-3}\left(1-y_{1}\right)\left(x+y_{3}-3 y_{2}\right)(x+$ $\left.y_{2}-3 y_{3}\right)+12 b a^{-3}\left(1-y_{1}\right)^{2}\left(x+y_{2}-3 y_{3}\right)+3 b^{2}\left(x+y_{2}-\right.$ $\left.3 y_{3}\right)^{2}\left(x+y_{3}-3 y_{2}\right)+6 b^{2} a^{-3}\left(1-y_{1}\right)\left(x+y_{2}-3 y_{3}\right)^{2}+8(1-$ $\left.\left.y_{1}\right)^{3}+b^{3}\left(x+y_{2}-3 y_{3}\right)^{3}\right]$,

$A_{2}=a^{3} c\left[8 b^{3}\left(1+y_{1}\right)^{3}+24 b^{2} a^{-3}\left(1+y_{1}\right)^{2}\left(x+y_{2}+y_{3}\right)+\right.$ $\left.24 b a^{-3}\left(1+y_{1}\right)\left(x+y_{2}+y_{3}\right)^{2}+8\left(x+y_{2}+y_{3}\right)^{3}\right]-a^{3} c[(x+$ $\left.y_{3}-3 y_{2}\right)^{3}+6 b a^{-3}\left(1-y_{1}\right)\left(x+y_{3}-3 y_{2}\right)^{2}+3\left(x+y_{3}-\right.$ $\left.3 y_{2}\right)^{2}\left(x+y_{2}-3 y_{3}\right)+12 b^{2} a^{-3}\left(1-y_{1}\right)^{2}\left(x+y_{3}-3 y_{2}\right)+$ $12 b a^{-3}\left(1-y_{1}\right)\left(x+y_{2}-3 y_{3}\right)\left(x+y_{3}-3 y_{2}\right)+3\left(x+y_{3}-\right.$ $\left.3 y_{2}\right)\left(x+y_{2}-3 y_{3}\right)^{2}+8\left(1-y_{1}\right)^{3} b^{3}+12 b^{2} a^{-3}\left(1-y_{1}\right)^{2}(x+$ $\left.\left.y_{2}-3 y_{3}\right)+6 b a^{-3}\left(1-y_{1}\right)\left(x+y_{2}-3 y_{3}\right)^{2}+\left(x+y_{2}-3 y_{3}\right)^{3}\right]$, $A_{3}=\left[b^{3}\left(x+y_{3}-3 y_{2}\right)^{3}+6 a^{-3} b^{2}\left(1-y_{1}\right)\left(x+y_{3}-3 y_{2}\right)^{2}+\right.$ $3 b^{2}\left(x+y_{3}-3 y_{2}\right)^{2}\left(x+y_{2}-3 y_{3}\right)+8\left(1-y_{1}\right)^{3}+12 b a^{-3}(1-$ $\left.y_{1}\right)\left(x+y_{3}-3 y_{2}\right)\left(x+y_{2}-3 y_{3}\right)+3 b\left(x+y_{3}-3 y_{2}\right)(x+$ $\left.y_{2}-3 y_{3}\right)^{2}+12 b a^{-3}\left(1-y_{1}\right)^{2}\left(x+y_{3}-3 y_{2}\right)+12 a^{-3}(1-$ $\left.y_{1}\right)^{2}\left(x+y_{2}-3 y_{3}\right)+6 a^{-3}\left(1-y_{1}\right)\left(x+y_{2}-3 y_{3}\right)^{2}+\left(x+y_{2}-\right.$ $\left.\left.3 y_{3}\right)^{3}\right]-\left[8\left(1+y_{1}\right)^{3}+12 a^{-3}\left(1+y_{1}\right)^{2}(b+1)\left(x+y_{2}+y_{3}\right)+\right.$ $\left.6 a^{-3}\left(1+y_{1}\right)(b+1)^{2}\left(x+y_{2}+y_{3}\right)^{2}+(1+b)^{3}\left(x+y_{2}+y_{3}\right)^{3}\right]$, $A_{4}=\left[b^{3}\left(x+y_{3}-3 y_{2}\right)^{3}+6 a^{-3} b^{2}\left(1-y_{1}\right)\left(x+y_{3}-3 y_{2}\right)^{2}+\right.$ $3 b^{2}\left(x+y_{3}-3 y_{2}\right)^{2}\left(x+y_{2}-3 y_{3}\right)+8\left(1-y_{1}\right)^{3}+12 b a^{-3}(1-$ $\left.y_{1}\right)\left(x+y_{3}-3 y_{2}\right)\left(x+y_{2}-3 y_{3}\right)+3 b\left(x+y_{3}-3 y_{2}\right)\left(x+y_{2}-\right.$ $\left.3 y_{3}\right)^{2}+12 b a^{-3}\left(1-y_{1}\right)^{2}\left(x+y_{3}-3 y_{2}\right)+12 a^{-3}\left(1-y_{1}\right)^{2}(x+$ $\left.\left.y_{2}-3 y_{3}\right)+6 a^{-3}\left(1-y_{1}\right)\left(x+y_{2}-3 y_{3}\right)^{2}+\left(x+y_{2}-3 y_{3}\right)^{3}\right]-$ $\left[\left(x+y_{3}-3 y_{2}\right)^{3}+6 a^{-3}\left(1-y_{1}\right)\left(x+y_{3}-3 y_{2}\right)^{2}+12 a^{-3}(1-\right.$ $\left.y_{1}\right)^{2}\left(x+y_{3}-3 y_{2}\right)+3 b\left(x+y_{3}-3 y_{2}\right)^{2}\left(x+y_{2}-3 y_{3}\right)+$ $12 b a^{-3}\left(1-y_{1}\right)\left(x+y_{3}-3 y_{2}\right)\left(x+y_{2}-3 y_{3}\right)+12 b a^{-3}(1-$ $\left.y_{1}\right)^{2}\left(x+y_{2}-3 y_{3}\right)+3 b^{2}\left(x+y_{2}-3 y_{3}\right)^{2}\left(x+y_{3}-3 y_{2}\right)+$ $\left.6 b^{2} a^{-3}\left(1-y_{1}\right)\left(x+y_{2}-3 y_{3}\right)^{2}+8\left(1-y_{1}\right)^{3}+b^{3}\left(x+y_{2}-3 y_{3}\right)^{3}\right]$, $D=a^{3} c\left[8 b^{3}\left(1+y_{1}\right)^{3}+24 b^{2} a^{-3}\left(1+y_{1}\right)^{2}\left(x+y_{2}^{+} y_{3}\right)+\right.$ $\left.24 b a^{-3}\left(1+y_{1}\right)\left(x+y_{2}+y_{3}\right)^{2}+8\left(x+y_{2}+y_{3}\right)^{3}\right]+a^{3} c[(x+$ $\left.y_{3}-3 y_{2}\right)^{3}+6 b a^{-3}\left(1-y_{1}\right)\left(x+y_{3}-3 y_{2}\right)^{2}+3\left(x+y_{3}-\right.$ $\left.3 y_{2}\right)^{2}\left(x+y_{2}-3 y_{3}\right)+12 b^{2} a^{-3}\left(1-y_{1}\right)^{2}\left(x+y_{3}-3 y_{2}\right)+$ $12 b a^{-3}\left(1-y_{1}\right)\left(x+y_{2}-3 y_{3}\right)\left(x+y_{3}-3 y_{2}\right)+3\left(x+y_{3}-\right.$ $\left.3 y_{2}\right)\left(x+y_{2}-3 y_{3}\right)^{2}+8\left(1-y_{1}\right)^{3} b^{3}+12 b^{2} a^{-3}\left(1-y_{1}\right)^{2}(x+$ $\left.\left.y_{2}-3 y_{3}\right)+6 b a^{-3}\left(1-y_{1}\right)\left(x+y_{2}-3 y_{3}\right)^{2}+\left(x+y_{2}-3 y_{3}\right)^{3}\right]$.

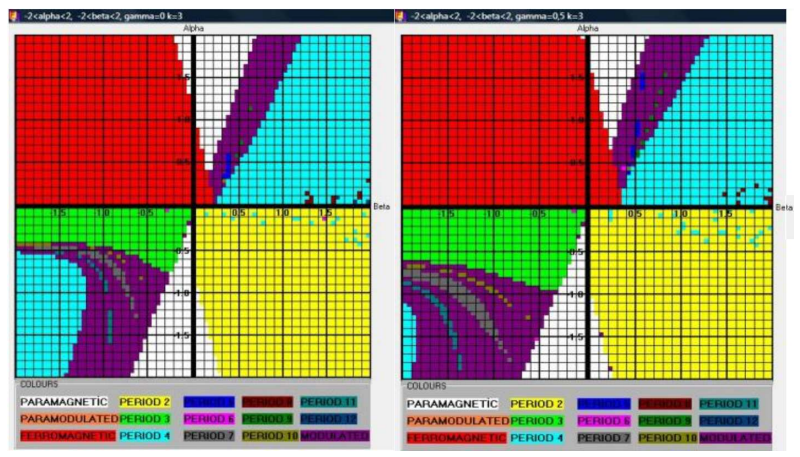

Fig. 1. The phase diagrams of the model for $k=3$, $\gamma=0$ (left) and $\gamma=0.5$ (right), respectively. 


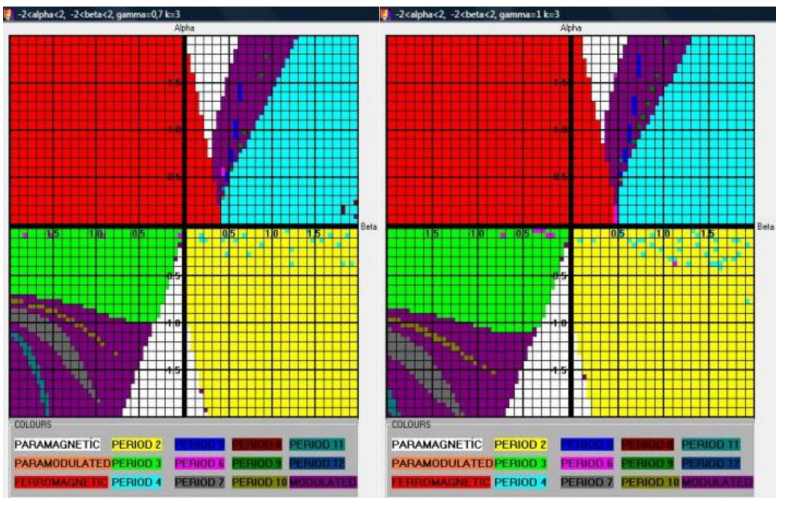

Fig. 2. The phase diagrams of the model for $k=3$, $\gamma=0.7$ (left) and $\gamma=1$ (right), respectively.
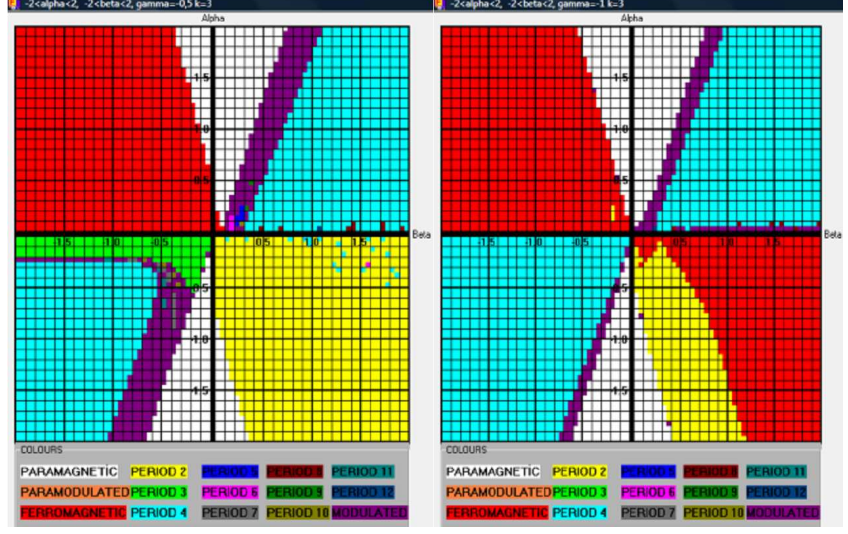

Fig. 3. The phase diagrams of the model for $k=3$, $\gamma=-0.5$ (left) and $\gamma=-1$ (right), respectively.

The system of four equations finally obtained (5) is essentially complicated than the similar basic equations $(1,3,4)$. Analytic investigation of Eq. (5) will be discussed later on. Below we use numerical methods to study its detailed behavior. The recursion relations (5) provide us the numerically exact phase diagram in $\left(T / J_{n n}\right.$, $\left.-J_{p n} / J_{n n}, J_{t t} / J_{n n}\right)$ space. Let $T / J_{n n}=\alpha,-J_{p n} / J_{n}=$ $\beta, J_{t t} / J_{n n}=\gamma$ and respectively $c=\exp \left(\alpha^{-1}\right), b=$ $\exp \left(-\alpha^{-1} \beta\right)$ and $a=\exp \left((3 \alpha)^{-1} \gamma\right)$. Starting from initial conditions

$$
\begin{aligned}
& \bar{\sigma}^{(n)}\left(\frac{V}{V_{n}}\right) \bar{\sigma}^{(n)}\left(\frac{V}{V_{n}}\right) \equiv 1, \\
& x^{(1)}=\frac{2 b^{2}+a^{2} c^{2}+1}{a^{3} c^{3} b^{2}+a c}, \quad y_{1}^{(1)}=\frac{a^{2} c^{2} b^{2}-1}{a^{2} c^{2} b^{2}+1}, \\
& y_{2}^{(1)}=\frac{b^{2}-a^{2} c^{2}}{a^{3} c^{3} b^{2}+a c}, \quad y_{3}^{(1)}=\frac{a^{2} c^{2}-1}{a^{3} c^{3} b^{2}+a c},
\end{aligned}
$$

one iterates the recurrence relations (5) and observes their behavior after a large number of iterations. In the simplest situation a fixed point $\left(x^{*}, y_{1}^{*}, y_{2}^{*}, y_{3}^{*}\right)$ is reached.
After a large number of iterations, if the fixed point is satisfied as $y_{1}^{*}=0, y_{2}^{*}=0, y_{3}^{*}=0$, then it corresponds to a paramagnetic phase. If $y_{1}^{*}, y_{2}^{*}, y_{3}^{*} \neq 0$, the phase diagram corresponds to the ferromagnetic phase. Similarly, we obtain the other phase regions. For the other cases, see the Ref. [6-9].

\section{Conclusions}

In this paper, we have studied the phase diagrams of the Potts model on the Cayley tree of order three. In order to calculate the recurrences equations are much more difficult than the two order case. Until now, the diagrams of Potts have been investigated for the Cayley tree of order two [6]-[9]. In this case, we have seen that the phase diagrams mainly have changed. We have observed that the prolonged interaction has strong effects on the phase diagrams. The Potts model on the Cayley tree of third order, with competing interactions between the nearestneighbor, prolonged next-nearest neighbor and two-level triple neighbors have been studied. The diagrams consist of six phases: ferromagnetic, paramagnetic, phase with period $p=2$ that corresponds to antiferromagnetic phase, with period $p=4$ that corresponds to so-called antiphase, with period $p=3$ and modulated phase with $p>12$. We have obtained some similar phase diagrams as [8], but some of figures obtained from [8] are different with respect to $\gamma$. If we select negative $\gamma$ then the period $p=4$ arises in the first and the third quadrants. In case of positive $\gamma$, in the third quadrant, if $\gamma$ increases, the period 4 decreases. In the next studies the magnetization and Lyapunov exponents will be investigated with the help of a recursion relation technique.

\section{Acknowledgment}

The work is supported by the Scientific and Technological Research Council of Turkey-TUBITAK (project No: 109T678).

\section{References}

[1] P. Bak, Rep. Prog. Phys. 45, 587 (1982).

[2] R.J. Elliott, Phys. Rev. 124, 346 (1961).

[3] S. Ostlund, Phys. Rev. B 24, 398 (1981).

[4] D.A. Huse, Phys. Rev. B 24, 5180 (1981).

[5] J. Vannimenus, Z. Phys. B 43, 141 (1981).

[6] N. Ganikhodjaev, F.M. Mukhamedov, C.H. Pah, Phys. Lett. A 373, 33 (2008)

[7] N. Ganikhodjaev, S. Temir, H. Akin, J. Stat. Phys. 137, 701 (2009)

[8] S. Temir, N. Ganikhodjaev, H. Akin, S. Uguz, AIP Conf. Proc. 1281, 2069 (2010)

[9] N. Ganikhodjaev, S. Temir, H. Akın, S. Uguz, J. Korean Phys. Soc. 59, 2971 (2011) 